\title{
Pyrosequencing Investigation into the Bacterial Community in Permafrost Soils along the China-Russia Crude Oil Pipeline (CRCOP)
}

\author{
Sizhong Yang ${ }^{1 *}, X_{i} W^{2}{ }^{2}$, Huijun Jin ${ }^{1}$, Qingbai $W^{1}$ \\ 1 State Key Laboratory of Frozen Soil Engineering (SKLFSE), Cold and Arid Regions Environmental and Engineering Research Institute (CAREERI), Chinese Academy of \\ Sciences, Lanzhou, China, 2 College of Electrical Engineering, Northwest University for Nationalities, Lanzhou, China
}

\begin{abstract}
The China-Russia Crude Oil Pipeline (CRCOP) goes through $441 \mathrm{~km}$ permafrost soils in northeastern China. The bioremediation in case of oil spills is a major concern. So far, little is known about the indigenous bacteria inhabiting in the permafrost soils along the pipeline. A pilot 454 pyrosequencing analysis on the communities from four selected sites which possess high environment risk along the CRCOP is herein presented. The results reveal an immense bacterial diversity than previously anticipated. A total of 14448 OTUs with 84834 reads are identified, which could be assigned into 39 different phyla, and 223 families or 386 genera. Only five phyla sustain a mean OTU abundance more than $5 \%$ in all the samples, but they altogether account for $85.08 \%$ of total reads. Proteobacteria accounts for $41.65 \%$ of the total OTUs or $45 \%$ of the reads across all samples, and its proportion generally increases with soil depth, but OTUs numerically decline. Among Proteobacteria, the abundance of Beta-, Alpha-, Delta- and Gamma- subdivisions average to 38.7\% (2331 OTUs), $37.5 \%$ (2257 OTUs), 10.35\% (616 OTUs), and 6.21\% (374 OTUs), respectively. Acidobacteria (esp. Acidobacteriaceae), Actinobacteria (esp. Intrasporangiaceae), Bacteroidetes (esp. Sphingobacteria and Flavobacteria) and Chloroflexi (esp. Anaerolineaceae) are also very common, accounting for $8.56 \%$ (1237 OTUs), 7.86\% (1136 OTUs); 7.35\% (1063 OTUs) and $8.27 \%$ (1195 OTUs) of total libraries, respectively. The ordination analysis indicates that bacteria communities in the upper active layer cluster together (similar), while bacterial consortia from the lower active layer and permafrost table scatter (less similar). The abundance of Rhodococcus (12 OTUs), Pseudomonas (71 OTUs) and Sphingomonas (87 OTUs) is even less ( $<0.01 \%)$. This effort to profile the background diversity may set the first stage for better evaluating the bacterial dynamics in response to accidental oil spills.
\end{abstract}

Citation: Yang S, Wen X, Jin H, Wu Q (2012) Pyrosequencing Investigation into the Bacterial Community in Permafrost Soils along the China-Russia Crude Oil Pipeline (CRCOP). PLOS ONE 7(12): e52730. doi:10.1371/journal.pone.0052730

Editor: Michael Watson, The Roslin Institute, University of Edinburgh, United Kingdom

Received July 5, 2012; Accepted November 21, 2012; Published December 26, 2012

Copyright: ( 2012 Yang et al. This is an open-access article distributed under the terms of the Creative Commons Attribution License, which permits unrestricted use, distribution, and reproduction in any medium, provided the original author and source are credited.

Funding: This work is supported by the National Natural Science Foundation of China (NSFC) (Grant No. 20091044) (http://isisn.nsfc.gov.cn/egrantweb/). The funders had no role in study design, data collection and analysis, decision to publish, or preparation of the manuscript.

Competing Interests: The authors have declared that no competing interests exist.

*E-mail: yangsz@|zb.ac.cn

\section{Introduction}

The latitudinal permafrost in northeastern China $\left(0.38 \times 10^{6} \mathrm{~km}^{2}\right)$ is mainly distributed in the northern part of the Da and Xiao Xing'an Mountains. The permafrost zone has strong spatial heterogeneity due to the comprehensive geological elements of rugged diverse terrains and complex ecological environments in the northeastern China. As located in the southeast margin of Eurasian cryolithozone, the permafrost body is relatively warmer and is significantly more sensitive to climate change compared to those in Siberia and Alaska. The warming climate has accelerated the degradation of permafrost in past decades, as evidenced by the reduced thickness of permafrost and the increased depths of seasonal thaw, increased ground temperatures, and prolonged northward retreat of the southern limit of permafrost (SLP) [1]. Furthermore, the increasing anthropogenic activities (e.g., agriculture and linear infrastructures) since the 1950s further disturbed and fragmented the permafrost environment.

In 2010, the 953-km-long China-Russia Crude Oil Pipeline (CRCOP) was built with a modified conventional burial construction mode. It crosses $441 \mathrm{~km}$ of discontinuous permafrost and $465 \mathrm{~km}$ of deep $(>1.5 \mathrm{~m})$ seasonally frozen ground [2]. Since then, the CRCOP has been the focus of cold regions environment and engineering practices. For pipelines in cold regions, oil spills normally occur in the early commercial operation due to the differential frost-heave and thaw-settlement, and in the late stages because of cumulative corrosion and aging [3]. Oil spills can cause severe damage to cold ecosystems. For example, at least five accidents in Usinsk in Northern Russia have taken place since 1986, causing very serious adverse environmental impacts [3]. Moreover, the same levels of contamination can heavily damage the cold environments, as the cold ecosystems have adapted to harsh conditions in ways that make them more fragile [4].

Globally, bioremediation is a good choice for remediating petroleum hydrocarbon contaminants because it is more environmentally friendly and cost-effective than other alternative, generally more energy intensive approaches. Many indigenous cold-adapted microbial populations have been observed to be capable to remediate hydrocarbons-contaminated soils at low temperature (e.g. [5-8]). Microbial degraders could get enriched from less than $0.1 \%$ of total bacterial population in pristine environments to up to $100 \%$ of the population [9]. Hydrocarbons 
ranging from $\mathrm{C}_{10}$ to $\mathrm{C}_{26}$ and aromatics of low molecular weight can be readily degraded, while more complex molecular structures are generally more resistant to biodegradation [10].

Bioremediation research in China has been enhanced in recent years. Microbial degraders were isolated from polluted soils in Liaoyang, Dagang [11-13], Shengli oil fields [14] and wetlands in the Liaodong Bay of China [15]. Some studies investigated the soil microbial diversity in Huabei, Dagang Oil fields in North China $[16,17]$, and community changes in response to contaminants in Changqing oil field [18], Daqing [19] and Yellow River Delta [20]. Some pilot experiments were also carried out on oil field soils at the bench scale [12,21] and field scale [22]. In addition, bioaugmentation technique was also tested in Dalian Bay recently [23]. Generally, almost all of these works focus on temperate oil fields where spills came out after long-time exploration.

So far, little is known about hydrocarbon degrading bacteria in the permafrost soils in the northeastern China. According to the environmental impact statement, high risks for oil leakage should be anticipated in segments along the CRCOP with the complicated engineering geology, particularly when the pipeline crosses the natural wetland reserves or important water supply sources [24]. The bioremediation of hydrocarbon pollutants is therefore very practical and necessary. This pipeline provides an opportunity to investigate the microbial degradation in case of oil spills. Current research on this subject is still in infancy. The knowledge about specific indigenous microbes, genes, and enzymes involved in hydrocarbon biodegradation within the permafrost environments along the pipeline is still scarce.

Considerable numbers and biodiversity of bacteria has been found in permafrost soils [25]. A global survey of 16S rRNA libraries indicated that Proteobacteria and Acidobacteria comprised roughly $40 \%$ and $20 \%$ of the communities, and Chloroflexi and Firmicutes were also abundant $(>5 \%$ of the community) in a wide range of soil environments [26]. Molecular techniques revealed the dominance of Gammaproteobacteria (esp. Xanthomonadaceae, 75-84\%) and Actinobacteria (39-57\%) in Siberian permafrost, and Grampositives (up to $45 \%$ ) and Proteobacteria (up to 25\%) in Antarctic permafrost $[27,28]$. Canadian Arctic permafrost is dominated by members of Proteobacteria, Actinomycetes, Firmicutes, Planctomyces, CFB, and Gemmatimonadetes [25]. Some groups are very important to carbon turnover. The dominant methane-oxidizing Proteobacteria (MOP) is affiliated with the Gamma- (type I) and Alpha- proteobacteria (type II) [27].

Conventionally, the biodiversity of permafrost microbes can be rapidly profiled by the DNA fingerprint methods, but the patterns of abundance and co-occurrence depend on the resolution of analytical methods [29]. Recently, pyrosequencing showed promise to capture the microbial taxa especially the low-abundant species. It can eliminate the laborious step of producing clone libraries and generate large number of sequences in a single run [30]. This work reported our pioneer research in high-throughput sequencing, and the results reveal an immense bacterial diversity in the active layer and permafrost table at four sites along the CRCOP pipeline. More importantly, this work may help explore the natural bioremedial consortia in the permafrost soils along the CRCOP.

\section{Research Sites}

The permafrost along the GRCOP is in the south margin of the latitudinal permafrost in East Asia [2]. The pipeline crosses both seasonally frozen ground and permafrost regions, where discontinuous, sporadic and isolated patches of permafrost were developed on the basis of varied terrains, soil textures, water contents, slope aspects and vegetative coverage. The heterogeneous conditions distinguished the CRCOP from the Alyeska Pipeline system and Norman Wells Pipeline. The complex permafrost conditions have greatly complicated the design and construction of the CRCOP pipeline [2]. According to the environmental assessment, high-risk of spills were considered in the 157 sections where pipeline cross the transition area with different permafrost conditions [24].

In this study, four research sites were selected along the pipeline, which potentially represent four permafrost subzones prone to oil spills (Figure 1, referring details to Jin et al. [2]). All sites stand on permafrost-affected bogs, but differ in area extent, soil temperature and permafrost thickness. Microbial soil samples were collected during the pipeline trench excavation in winter of 2009. For each profile, triplicate samples were retrieved from layers 1) the upper active layer (normally $30-40 \mathrm{~cm}$, below the modern grass rhizosphere), 2) the lower (deep) active layers (depth depending on the sites), and 3) the permafrost table. The soil samples were collected in the state-owned land which is open for scientific research. No specified permissions are required for these sampling sites, which are not natural reserve and did not involve endangered or protected species.

\section{Methods}

\section{DNA extraction, PCR and pyrosequencing}

Soil DNA was extracted using commercial Power Soil DNA Isolation Kit (MOBIO, USA) according to the instruction. The triplicate DNA extracts for each layer were pooled prior to downstream manipulation. Universal bacterial primer set $8 \mathrm{~F}\left(5^{\prime}-3^{\prime}\right.$ GAGTTTGATCGTGGCTCAG) and 533R (5'-3' TTACGGCGGCTGCTGGCAC) covering V1-V3 regions of SSU were synthesized by Shanghai Majorbio Bio-pharm Technology Co., Ltd. (Majorbio as below). Different barcode sequences were added at the $5^{\prime}$ end of the forward primer for multiplexed pyrosequencing. PGR were carried out in a $20 \mu \mathrm{L}$ reaction volumes containing $0.5 \mathrm{ul}$ DNA template, $250 \mu \mathrm{M}$ dNTPs, $0.1 \mu \mathrm{M}$ of each primer and 2.5 U FastPfu Polymerase (Applied Biosystems) in the appropriate $5 \times$ FastPfu Buffer and de-ionized ultrapure water. The protocol was optimized with low cycles for better accuracy and reliability of the subsequent data analysis. The PCR condition were initial denaturation at $95^{\circ} \mathrm{C}$ for $2 \mathrm{~min}$, followed by 25 cycles of denaturation at $94^{\circ} \mathrm{C}$ for $30 \mathrm{~s}$, annealing at $55^{\circ} \mathrm{C}$ for $30 \mathrm{~s}$ and extension at $72^{\circ} \mathrm{C}$ for $30 \mathrm{~s}$, with a final extension phase at $72^{\circ} \mathrm{C}$ for $5 \mathrm{~min}$. PCR products $(3 \mu \mathrm{L})$ were checked on a $2 \%$ agarose gel. PCR products were purified using MiniElute PCR purification kit (Qiagen) and quantified using the GeneQuant pro system. Samples were then pooled at equal concentrations. Parallel tagged sequencing was performed using a Roche 454 GS FLX in Majorbio.

\section{Bioinformatic analysis}

Data preprocessing was performed mainly upon software of mothur [31]. The raw sequence was trimmed off the standard primers and barcodes, assembled the reads to contigs. Sequences less than $150 \mathrm{bp}$ in length and greater than 3\% low quality bases (quality score $<27$ ) were removed. The chimeric sequences were also excluded by the chimera.uchime command with default parameters. These valid sequences were finally trimmed to $300 \mathrm{bp}$ and then aligned with needleman algorithm and clustered with the bacterial SILVA database (SILVA 108). The candidate sequences were assigned to the taxonomy with classify.seqs command (Bayesian approach). And the dist.seqs command generated the distance matrix between aligned DNA sequences. Gap compar- 


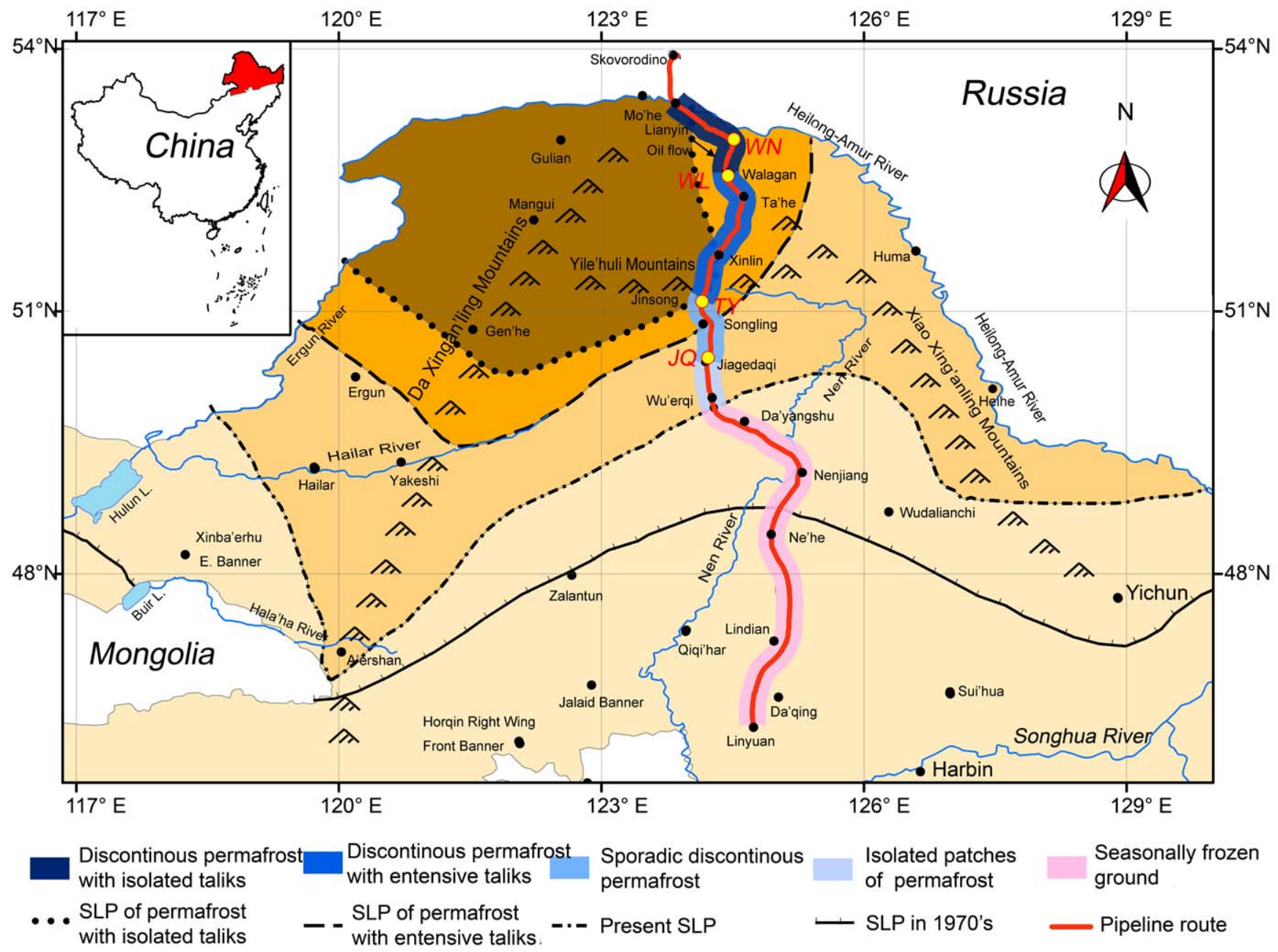

Figure 1. Schematic map showing the sampling sites along the China-Russia Crude Oil Pipeline route (after Jin et al. [2]). WN: Walagan north; WL: Walagan; TY: Tayuan; JQ: Jiagedaqi; SLP: Southern limit of permafrost.

doi:10.1371/journal.pone.0052730.g001

isons and terminal gaps were handled with the method option of calc $=$ onegap and countends $=\mathrm{T}$. Then, these sequences were clustered to OTUs (operational taxonomic units) at $97 \%$ sequence identity by using mothur (furthest neighbor method) and chopseq (Majorbio). Rarefaction analysis was performed by mothur and plot-rarefaction (Majorbio). From these, the Shannon diversities and the Chaol richness estimations were calculated by mothur. The weighted UniFrac distance was used to quantify differences in community composition. Heatmap figure and Venn diagrams were implemented by $R$ packages pheatmap [32] and VennDiagram [33], respectively. In addition, weighted principal component analysis (PCA) and Nonmetric Multidimensional Scaling (NMDS) diagrams were generated by using $R$ package vegan [34] to demonstrate the clustering of different samples. The sequences for this article have been deposited in NCBI SRA under the accession number SRA057910.

\section{Results}

A total of 84834 reads and 14448 OTUs were obtained from the 12 samples through 454 pyrosequencing analysis. Each library contains 5600 to 8117 reads, with different phylogenetic OTUs ranging from 566 to 2687 . The rarefaction curves tend to approach the saturation plateau except in the samples of 1300 and
3300 (Figure 2). The Good's coverage index reveals that $90 \%$ to $96 \%$ of the species were obtained in eight samples, and $85-87 \%$ for samples of 2500 and 2300, while there are still some fraction of species diversity remains to be discovered in the samples of 1300 $(68 \%)$ and $3300(74 \%)$ respectively. The samples from upper active layer (e.g., the samples of 1300, 2300, 3300 and 4300) were plotted in the upper part in Figure 2. This rarefaction curve indicates a large variation in the total number of OTUs in different samples, but the sequence coverage is still sufficient to capture the diversity of the bacterial communities, whereas the OTUs density is larger in the upper layer than the lower parts.

\section{Taxonomic composition}

Out of the twelve samples they comprised of different numbers of OTUs and OTU abundances. Sequences that could not be classified into any known group are assigned as $\mathcal{N}_{0} \_$Rank. These bacterial OTUs can be assigned into 39 different phyla, 223 families or 386 genera. Thirteen different phyla out of the 39 total phylotypes are common to the whole 12 libraries, which occupy $97.53 \%, 96.38 \%, 95.32 \%, 93.92 \%$ of the total reads in the libraries of Walagan (WL), Walagan North (WN), Tayuan (TY) and Jiagedaqi (JQ), respectively. Only five phyla comprise mean OTUs abundance more than $5 \%$ in each sample, but they jointly 


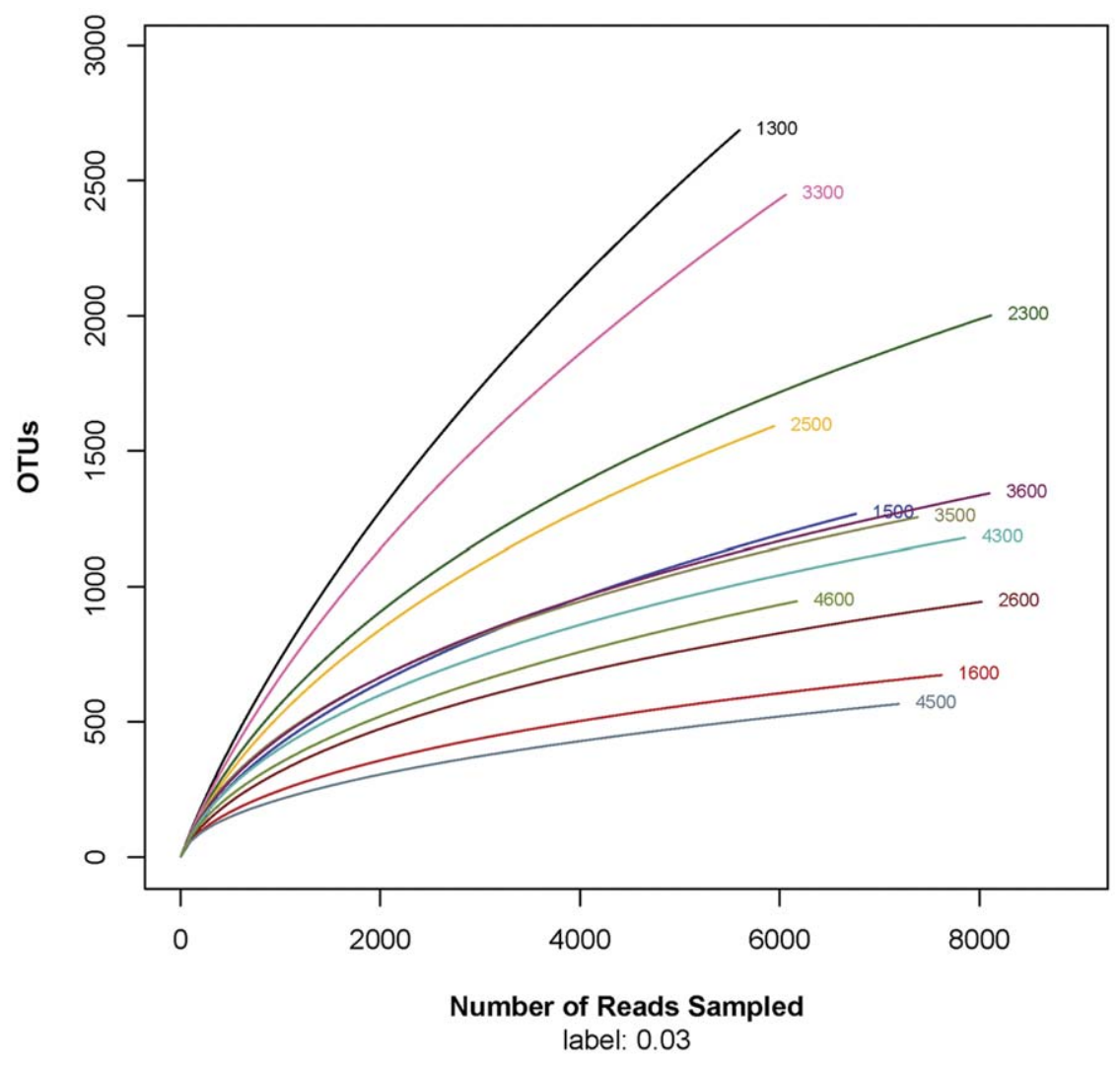

Figure 2. Rarefaction analysis of the different samples. Rarefaction curves of OTUs clustered at $97 \%$ phylotype similarity level. The sample labels beginning with numeric 1, 2, 3 and 4 correspond to the sampling locations of Walagan (WL), Walagan north (WN), Tayuan (TY) and Jiagedaqi (JQ). The second digit $(3,5,6)$ in the labels represents the upper, the lower active layer and the permafrost table, respectively. doi:10.1371/journal.pone.0052730.g002

hold $85.08 \%$ of the total reads. Proteobacteria is the most abundant division (Figure 2), comprising approximately $41.6 \%$ (6018) OTUs and $45.08 \%$ (38196) reads across all samples, whereas the members from Acidobacteria (8.56\%, 1237 OTUs), Actinobacteria (7.86\%, 1136 OTUs), Bacteroidetes (7.35\%, 1063 OTUs) and Chloroflexi $(8.27 \%, 1195$ OTUs) make up $8.20 \%$ (6955 reads), $8.16 \%$ (6922 reads), $6.38 \%$ (5413 reads) and $5.10 \%$ (4325 reads) of the whole libraries, respectively. The average reads of No_rank group accounts for $2.78 \%$, but fluctuates in different sites and depths. There are also a certain proportion of members of Firmicutes (10.37\%, 8801 reads; 4.94\%, 715 OTUs), Planctomycetes (0.95\%, 782 reads; 3.80\%, 549 OTUs), Verrucomicrobia $(1.65 \%$, 1401 reads; $1.75 \%, 253$ OTUs) and Nitrospirae (1.34\%, 1136 reads; $0.82 \%, 119$ OTUs). The other lineages represent much smaller fraction (ca. 7\%) of the bacterial communities.

At family level, there are 19 lineages among the total 223 families exist in all samples, but these subgroups account for $71.86 \%, 70.28 \%, 80.25 \%$ and $80.68 \%$ of the total reads in the four research sites, respectively. The top 5 families, by descending read abundance, are $N_{0}$ Rank $(32.35 \%)$, Oxalobacteraceae $(15.86 \%)$, Comamonadaceae $(9.90 \%)$, Flavobacteriaceae $(2.37 \%)$ and Nitrosomonadaceae $(2.09 \%)$. According to OTUs, the top 5 phylotypes are Comamonadaceae $(4.39 \%$, 635 OTUs), Oxalobacteraceae $(3.70 \%, 535$ OTUs), Sphingomonadaceae $(3.17 \%, 459$ OTUs), Caulobacteraceae (2.42\%, 350 OTUs), and Chitinophagaceae (1.89\%, 274 OTUs).

Generally, each site contains approximately the similar phyla, and all have shown higher phylotypic richness in the upper active layer than the lower layers (Table 1). For example, the WL profile descends from 31 bacterial phyla in the upper active layer (1300), to 24 in the lower active layer (1500), and till to 14 lineages in the permafrost table (1600). Similar changes can be found in WN. The TY and JQ profiles, however, show relatively lower richness in the lower active layer $(70-80 \mathrm{~cm})$. In addition, these bacterial consortia differ vertically in the relative proportions of different phyla in the whole libraries. A striking feature is that the portion of the dominant Proteobacteria readily increases but the number of OTUs decrease with depth in three research sites, especially in $\mathrm{WN}$ and $\mathrm{WL}$ which are located in the northern part of the pipeline route, while JQ displays its highest peak in the lower active layers. On the other hand, the TY profile demonstrates a generally descending trend (Figure 3). The second predominant phylum of Acidobacteria shows an increasing trend in WN, WL and JQ but declines in TY. The division of Bacteroidetes sustains relatively similar read abundance in the upper active layer and permafrost table, but a peak occurs in the deep active layer of the WL $(10 \%$ vs $6 \%)$ and $\mathrm{WN}(22 \%$ vs $13 \%)$, that is unlike the gradual decrease trend in profiles of TY and JQ. The other phyla show unsystematic vertical variations in comparison to the Proteobacteria and Acidobacteria.

The members of Betaproteobacteria dominate the Proteobacteria phylum, occupy $16.13 \%$ (2331 OTUs) of total, but take over $34.03 \%$ of the total reads (ranging from $17.63-75.58 \%$ ). The subdivision of Alphaproteobacteria sustains $15.62 \%$ (2257) of the total OTUs, and only an average of $6.10 \%$ of total reads but showing high variability $(0.64 \%-26.74 \%)$. Gammaproteobacteria and Deltaproteobacteria make up $2.58 \%$ (374 OTUs) and $4.26 \%$ (616 OTUs) in total phylotypes, and have $5.97 \%$ and $5.56 \%$ reads in the total, respectively. The Epsilonproteobacteria only contains 8 OTUs 


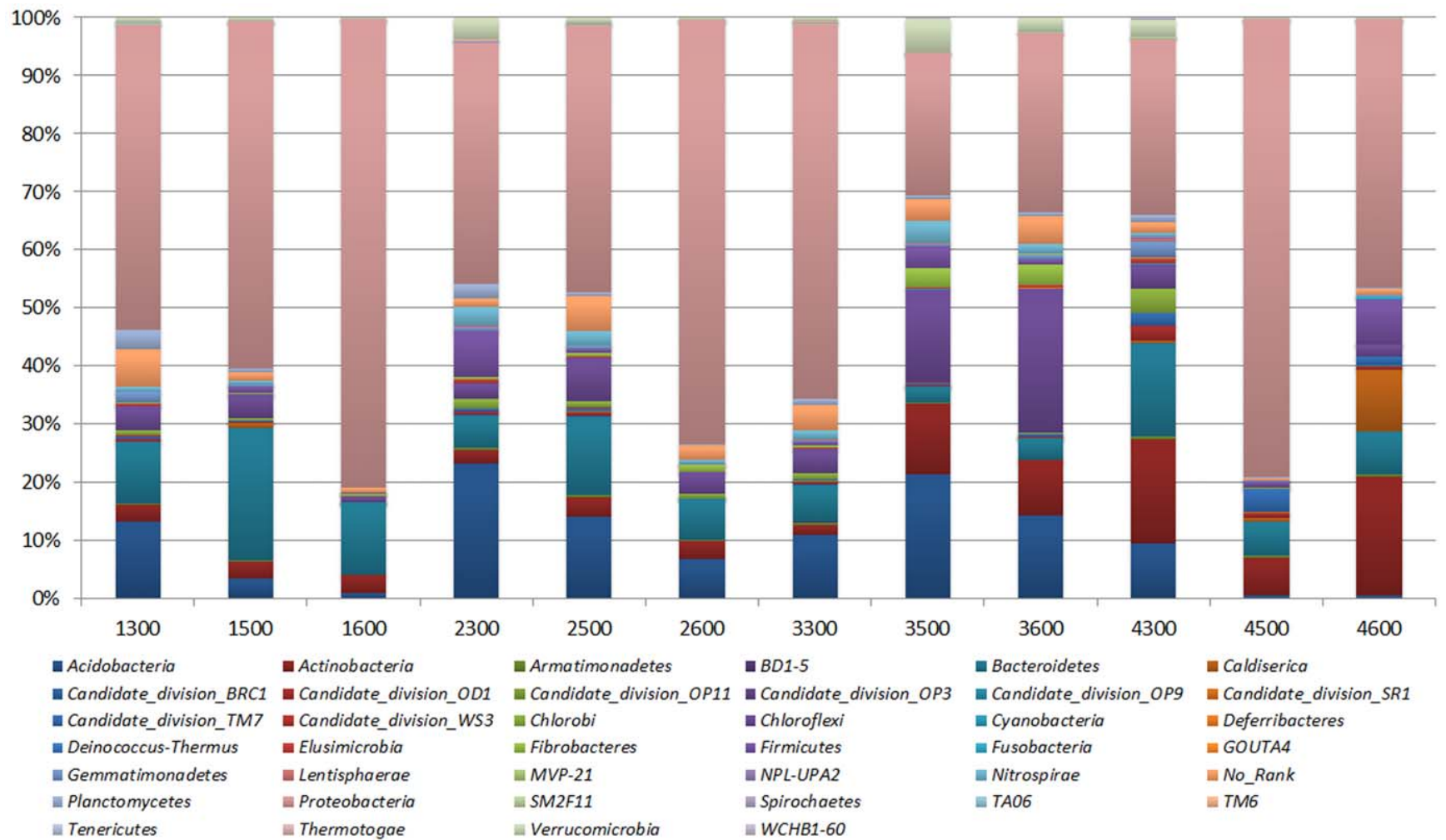

Figure 3. Bacterial composition of the different communities at the phylum level. Relative read abundance of different bacterial phyla within the different communities. Sequences that could not be classified into any known group were assigned as No_Rank.

doi:10.1371/journal.pone.0052730.g003

$(0.05 \%)$ and a very small fraction $(0.31 \%)$ in total reads. Statistically, the members of Betaproteobacteria contribute $38.73 \%$ to the total Proteobacteria reads, and Proteobacteria fluctuation is strongly correlated with the Betaproteobacteria (coefficient is 0.78 ) across all samples, particularly in the TY and JQ samples. At the permafrost table of WL (1600) and WN (2600), high abundant Alphaproteobacteria (34.94\%) and Deltaproteobacteria (23.12\%) further raise the read abundance of Proteobacteria. Among the Bacteroidetes, members of Sphingobacteria and Flavobacteria are most abundant, making up $3.62 \%$ and $2.41 \%$ accordingly in total reads. But vertically, Bacteroidetes is dominated by members of Chitinophagaceae $(1.55 \%)$ in the upper layer and Flavobacteriaceae $(3.77 \%)$ at the permafrost table. The members of Actinobacteria are less abundant in the upper active layer compared with permafrost table. Among Actinobacteria, the most common group is Intrasporangiaceae with $1.24 \%$ reads in the total libraries of the lower active layer and Micrococcaceae $(1.09 \%)$ at the permafrost table, respectively.

\section{Bacterial community variation with sites and depths}

The hierarchical heatmap (Figure 4) is based on the top 100 abundant bacterial community at family level, which generally indicates two groups. One is mainly composed of the upper layer samples $(1300,2300,4300)$; the other is chiefly clustered by the deep samples $(1500,1600,2600,3600,4500,4600)$. According to the heatmap, high similarity of the communities could be found in the same profile (e.g., lower active layer (4500) and permafrost table (4600) in JQ), or different sites (e.g., permafrost table samples of 1600 and 3600), or different layers in different sites (e.g., WN (2300) and TY (3500)). The principal component analysis (PCA) (Figure 5A) indicates that the upper active layer communities group together in the bottom left of the graph along PC1, whereas the communities in the lower active layer and the permafrost table scatter in different parts in the plot. Clearly, the upper active layer clusters are closer (similar) than those in the lower active layer (1500, 3500 and 4500) and the permafrost table (1600, 2600, 3600 and 4600). The PCA analysis agrees with the heatmap to indicate

Table 1. Vertical variations of bacterial phyla in the four research profiles.

\begin{tabular}{|c|c|c|c|c|c|c|c|c|}
\hline \multirow{2}{*}{ Horizon } & \multicolumn{2}{|l|}{ WN } & \multicolumn{2}{|l|}{ WL } & \multicolumn{2}{|l|}{ TY } & \multicolumn{2}{|l|}{ JQ } \\
\hline & Depth(cm) & phyla & Depth $(\mathrm{cm})$ & phyla & Depth(cm) & phyla & Depth(cm) & phyla \\
\hline Upper active layer & $30-40$ & 32 & $30-40$ & 31 & $30-40$ & 31 & $30-40$ & 30 \\
\hline Lower active layer & $70-80$ & 26 & $70-80$ & 24 & $80-90$ & 27 & $70-80$ & 26 \\
\hline Permafrost table & $130-140$ & 23 & $150-160$ & 14 & $120-130$ & 29 & $140-150$ & 29 \\
\hline Total observed & & 33 & & 33 & & 34 & & 35 \\
\hline
\end{tabular}


the far dissimilarity between 1500 and 3500, or high similarity between 2600 and 4600 libraries. The NMDS analysis based on the Bray-Curtis distance (Figure 5B), also confirms the more similar bacterial communities in the upper active layers than the other layers. The direction and position of the environmental factors, calculated with the envfit function in the $R$ vegan package [34], suggest the bacterial community in the upper active layers is somewhat related to the environmental parameters of total organic carbon (TOC), total phosphorus (TP) and nitrogen (TN), and water content. However, the correlation does not reach statistical significance.

The shared communities at different layers are further determined via the Venn diagram (Figure 6). In the upper active layer below the modern grass rhizosphere, a total of 52 families are shared by the four research sites (Figure 6A), accounting for $29.21 \%$ of the total 178 bacterial taxa in this layer. The top 10 abundant families shared by the four sites, according to the relative read abundance in the top 10 , are unclassified $\mathcal{N}_{0}$ _Rank groups (10969 reads, holding 39.69\% of the total), Betaproteobacteria (2792 reads), Alphaproteobacteria (772 reads, esp. 437 Xanthobacteraceae and 271 Bradyrhizobiaceae), Bacteroidetes (428 reads, esp. Chitinophagaceae, 428 reads), Gemmatimonadetes (374 reads, mostly Gemmatimonadaceae), Chloroflexi (372 reads, esp. Anaerolineaceae) and Planctomycetes (317 reads, esp. Planctomycetaceae). Among the Betaproteobacteria, the dominant members are assigned into Comamonadaceae (1530 reads), Nitrosomonadaceae (1007 reads), and Rhodocyclaceae (255 reads).

In the lower active layer, a total of 152 families of bacteria are identified, 35 of them $(23.02 \%)$ are common in all the four research sites (Figure 6B). Of the top 10 families, the Betaproteobacteria with average 9315 reads is the first dominant group, and it is mainly composed by members from Oxalobacteraceae (5400 reads, 19.79\%) and Comamonadaceae (3915 reads, 14.35\%). The No_Rank group, having 8514 reads $(31.20 \%)$, is also very common in this layer. Additionally, the libraries at the permafrost table, sharing 34 families, occupy $20.4 \%$ of the total 166 discovered taxa (Figure 6C). Betaproteobacteria is still predominant, taking up $33.68 \%$ of the total read abundance, which consists largely of members from Oxalobacteraceae (6621 reads, 22.13\%) and Comamonadaceae (2957 reads, 9.88\%). The No_Rank group is also abundant, containing $26.61 \%$ (7961 reads in total). Particularly, highly abundant Gammaproteobacteria is detected at the permafrost table, comprising a large proportion of Pseudomonadaceae (2850 reads, $9.53 \%$ ) and Moraxellaceae (850 reads, 2.84\%). Throughout the whole profiles, the bacterial communities in the upper layer are more diverse and shared more taxa than the other two layers.

\section{Discussion}

The permafrost along the CRCOP is overall warmer than that in Siberian Arctic, or contains higher carbon and water contents than that of the Tibetan Plateau, which may present a unique permafrost eco-niche in China. The 454 pyrosequencing in this study enable us to quickly capture both high and low-abundance phylotypes in a single run, to circumvent potential bias that conventional fingerprints and cloning procedures might introduce, and thus provide a different picture which can greatly improve our knowledge about the indigenous bacteria in permafrost soils along the CRCOP.

\section{Biodiversity background}

Based on our results, the permafrost soils along the CRCOP sustain an immense diversity of bacteria than previously expected. The predominant phyla are Proteobacteria (esp. Beta- and Alphasubdivision), Acidobacteria, Bacteroidetes, Actinobacteria, Chloroflexi,
Firmicutes, and Verrucomicrobia. The Chloroflexi sustains relatively high OTUs $(8.27 \%)$, but lower read abundance $(5.10 \%)$, while Firmicutes has low OTUs fraction (4.95\%) but high portion of reads (10.37\%). The typical peat-inhabiting Betaproteobacteria from acidic permafrost wetland are acidotolerant bacteria and can utilize various carbon substrates and fix $\mathrm{N}_{2}$ [35]. From this point, the acidic nature of the permafrost soil in this study is likely favorable for the members of Betaproteobacteria. This is unlike the pyrosequencing result of the arctic permafrost wetlands where the most common members are from Chloroflexi, Acidobacteria, and Actinobacteria (esp. Intrasporangiaceae and Rubrobacteraceae) [36]. The GFB groups with low members in this research are coincidence to those from the Siberia and Antarctic permafrost [28]. Recently, cultured representatives of Acidobacteria from cold peat bogs were found as acidophilic chemo-organotrophs that can grow at $\mathrm{pH}$ values between 3.0 and 6.5-7.5, and peat-inhabiting members of Actinobacteria, together with Verrucomicrobia, are less abundant but numerically significant groups of sequences in clone libraries in peat soil [35].

Surprisingly, the $\mathcal{N}_{0}$-Rank read abundance accounts for a large proportion $(>30 \%$ in average) in this study, a certain extent of candidate divisions are also detected. Similarly, unclassified bacteria could make up nearly $30 \%$ of clones in Arctic tundra soils, which probably play a significant yet unknown or less understood ecological role [28]. On the other hand, the 454 dataset offers a large suite of low-abundance communities with average fractions $<0.1 \%$, but they occupy $69 \%$ and $82 \%$ of total bacterial families and genus observed, accordingly. Similarly, lowabundance populations were revealed by pyrosequencing to greatly contribute to the phylogenetic diversity in deep water of the North Atlantic and hydrothermal vents [37]. Moreover, the members of Planctomycetes with low proportion are commonly observed in this study, were previously reported to be strongly underrepresented in the clone library from cold peatlands [35]. Since little is known about the global distribution of the rare populations, it is not yet possible to know whether they represent specific biogeographical distributions of bacterial taxa, or functional selection by particular permafrost environments.

Our dataset also indicated higher phylotypic richness is present in the upper active layer than lower layers, probably because the upper layer is in the oxic- anoxic interface, i.e., from the region of highest biological activity. The abundance of Proteobacteria generally increases with soil depth, mostly attributable to the subdivision of Betaproteobacteria. This is different from the result that Gammaproteobacteria members dominated in some anaerobic soils [38]. Another abundant group of Acidobacteria, often found in low $\mathrm{pH}$ soils [29], its abundance also tends to increase with depth in this study. Such increasing abundance was also identified in Siberia permafrost soils [25]. Generally, the microbial community difference is due to variations in permafrost environment [29]. For examples, the Siberian bacterial diversity in permafrost lower than the active layer is attributed to the low water availability, extremely low rates of nutrient exchange, and prolonged subfreezing temperature [25]. Soil redox conditions in permafrost were assumed to shape different structure of bacterial communities [39]. In addition, vegetation also exerts influences on bacterial community structure, e.g. in Alaskan soils [40]. In this study, the shifts in bacterial community structure are related, but not statistically to the degree of soil saturation, and the contents of TOC, TN and TP in the permafrost profile.

\section{Potential degraders}

Many of the bacteria capable of degrading oil are Gram negative and rod-shaped. Alkane-degrading bacteria from cold 


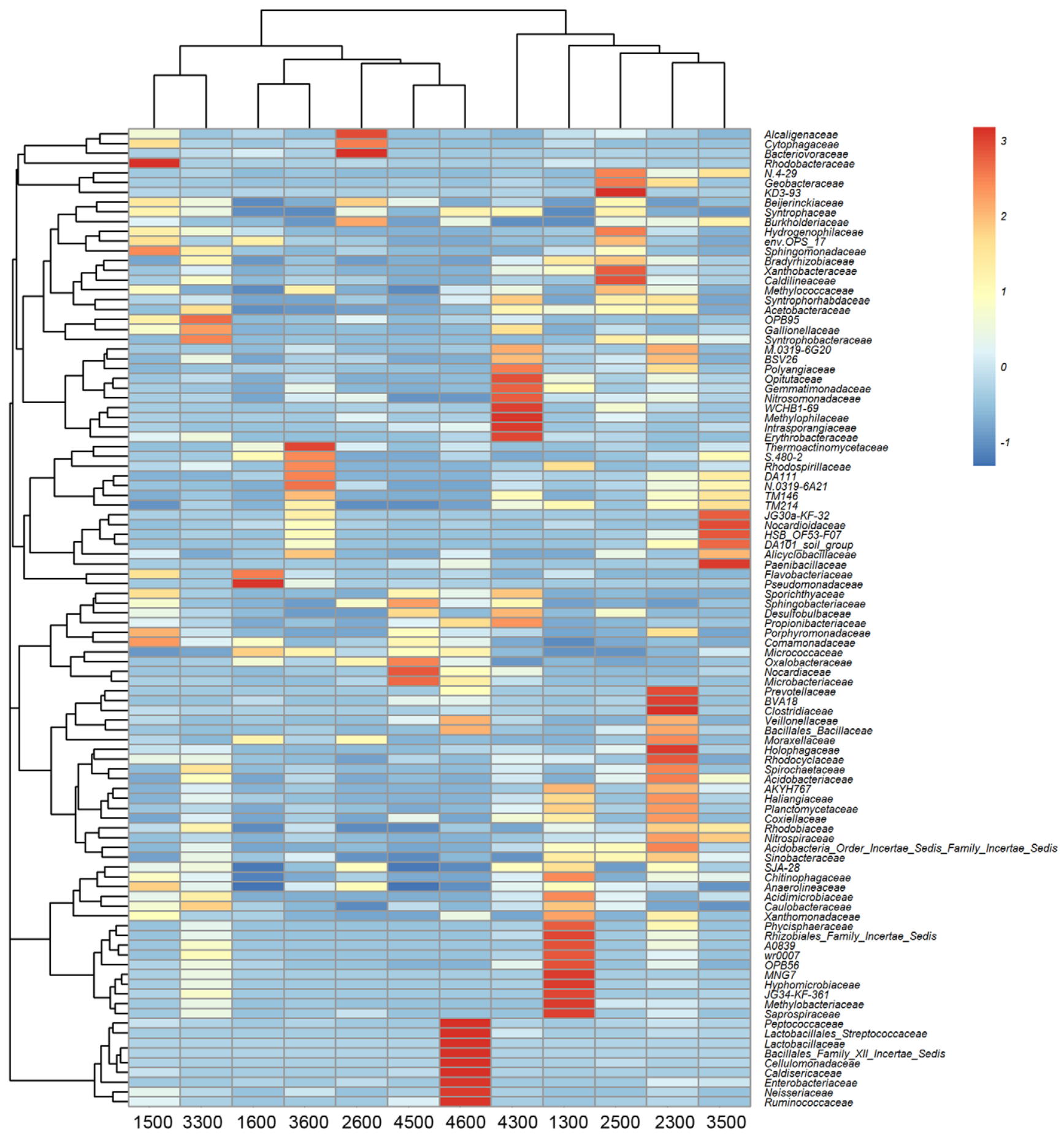

Figure 4. Bacterial distribution of the top $\mathbf{1 0 0}$ abundant families among the twelve samples. Double hierarchical dendrogram shows the bacterial distribution. The bacterial phylogenetic tree was calculated using the neighbor-joining method and the relationship among samples was determined by Bray-Curtis distance and the complete clustering method. The heatmap plot depicts the relative percentage of each bacterial family (variables clustering on the vertical-axis) within each sample (horizon-axis clustering). The relative values for bacterial family are indicated by color intensity with the legend indicated at the top right corner. doi:10.1371/journal.pone.0052730.g004

soils are frequently assigned to the Gram-positive genus Rhodococcus or the Gram-negative genus Pseudomonas; Aromatic-degrading bacteria from polar soils are typically classified into the Gramnegative bacterial genera Pseudomonas or Sphingomonas [10]. Except for the major groups, the biodegradation of alkanes were found correlated with groups related to denitrification, sulfate reduction from some years [41]. The Pseudomonas can degrade a narrower range of aromatic substrates than Sphingomonas spp. [10]. And, Acinetobacter from Gammaproteobacteria, contribute to the mineralization of aromatic compounds [42]. In our samples, Pseudomonas genus mostly exists in the deep active layer and permafrost table, with total OTUs fraction ca. $0.5 \%$, however, the read abundance 

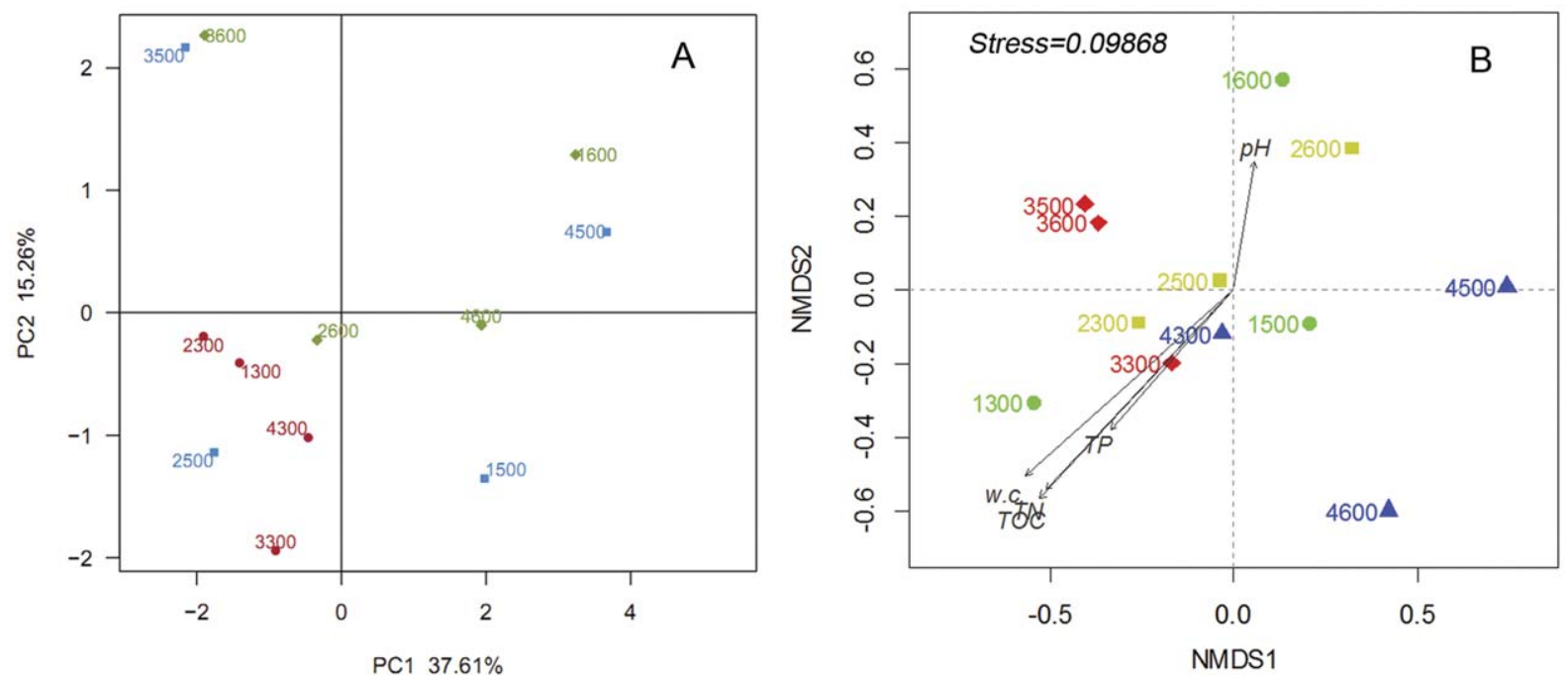

Figure 5. Sample sorting analysis. (A) Scatter plot of PCA-score showing similarity of the 12 bacterial communities based on Unifrac distance. Principal components (PCs) 1 and 2 explained $37.61 \%$ and $15.26 \%$ of the variance, respectively. (B) NMDs showing the difference of bacterial communities according to Bray-Curtis distance. TOC: total organic carbon, TN: total nitrogen, TP: total phosphorous, and w.c.: absolute water content (per gram dry soil).

doi:10.1371/journal.pone.0052730.g005

only occupies $0.3 \%$ (257 reads) of the total libraries. The Rhodococcus is a rare group across the 12 libraries, and only 12 OTUs are totally identified at genus level. Sphingomonas genus contains 87 OTUs but only 32 reads in the twelve samples. Among them, the potential degraders are expected to be explored. Some species in Flavobacterium genus capable of degrading pentachlorophenol $[43,44]$, are also observed in our samples, for example, 5 reads of Achromobacter and 1508 reads of Flavobacterium.

Populations of hydrocarbon-degraders normally constitute less than $1 \%$ of the indigenous microbial communities [45], which could be echoed by the groups less than $1 \%$ in this study. However, hydrocarbon contaminants can greatly shift both in quantity and composition [9]. Numbers of bacterial degraders can become elevated in contaminated soils to reach $10 \%$ to even $100 \%$ of the community [45], but the overall microbial diversity generally declines [10]. In Antarctic, hydrocarbon contamination could lead to enrichment of degrader of Proteobacteria (esp. genera Pseudomonas, Sphingomonas and Variovorax), and some Actinobacteria [46]. In the temperate Yellow River Delta in China, Alpha-, Beta- and some unknown Gamma- proteobacteria were enriched whereas Deltaproteobacteria, Firmicutes, Actinobacteria, Acidobacteria and Planctomycetes members were depleted by heavy crude spills [20]. The Gammaproteobacteria members could be enriched up to $30 \%$ of the total clone libraries observed in the seasonally frozen ground in Daqing, Northeast China, particularly members from Acinetobacter, then Arcobacter, Firmicutes, Pseudomonas, and Sulfurospirillum [47]. The strong shift towards the Gammaproteobacteria was also found in the Arctic sea-ice [48]. At present, the dynamic response of permafrost bacteria to the crude oil transported in the pipeline remains little known, but it would be the future focus.

The potential bioremediation can be affected by many factors. The number of active biodegraders certainly matters. Currently, total microbial numbers in these samples are generally at the magnitude of $10^{7}$ for indigenous consortia, and could quickly decline to $10^{3} \sim 10^{4}$ after 8 weeks in a lab contamination experiment (data not shown). A long period of incubation showed that degraders who are low or below detection limits in pristine polar soils could be enriched to more than $10^{5} \cdot \mathrm{g}^{-1}$ in contam-
A

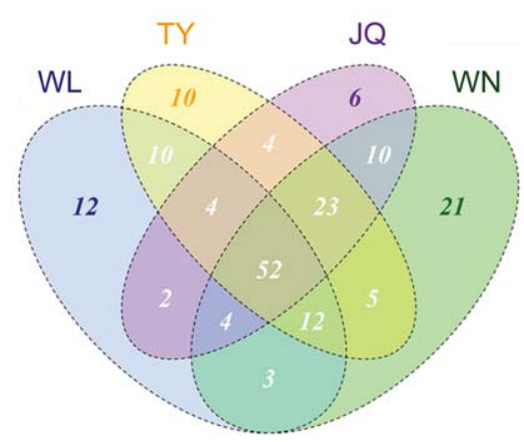

B

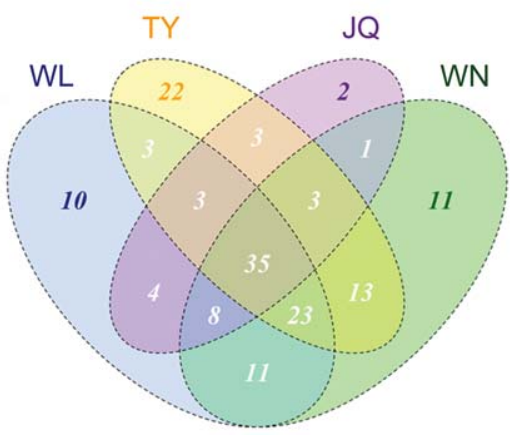

C

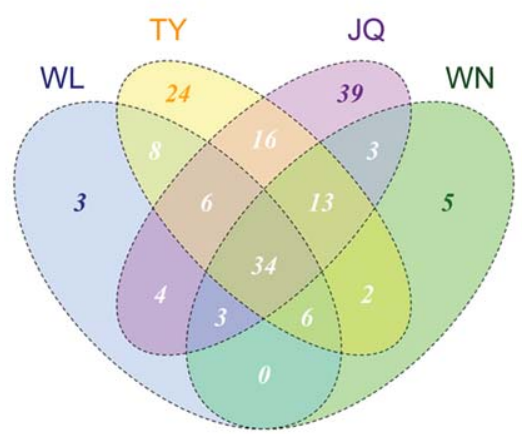

Figure 6. Venn diagram showing the unique and shared OTUs ( $3 \%$ distance level). (A) Upper active layer ( $30-40 \mathrm{~cm}$, including samples of 1300, 2300, 3300 and 4300), (B) Lower active layer (70-80 cm, including 1500, 2500, 3500 and 4500) and (C), Permafrost table (below $130 \mathrm{~cm}$, including 1600, 2600, 3600 and 4600) libraries. WL: Walagan, WN: Walagan North, TY: Tayuan, JQ: Jiagedaqi. doi:10.1371/journal.pone.0052730.g006 
inated soils in both surface and subsurface layers [49,50]. Other important factors include the composition of the oil, availability of nutrients and oxygen, water content, temperature, $\mathrm{pH}$ and salinity in cold regions [9]. Importantly, most microbial oil degradation strongly depends on the availability of oxygen, and nitrate or sulfate serving as a terminal electron acceptor [7]. In addition, temperature-water interaction can impact the bioremediation effectiveness due to their limitation to the oxygen availability in permafrost, especially during seasonal transition periods [10]. However, microbial degradation does not proceed linearly over time, it generally slows as the more readily degradable components are used up, leaving behind the more recalcitrant components [49].

\section{Potential in carbon turnover}

In the Da Xing'an Mountains, about 12\% surface is covered by permafrost-affected-peat bogs with average peat layer of $0.5-1 \mathrm{~m}$ [51]. The total soil organic carbon content averages to $30 \%$ in the active layer and $10 \%$ in the vicinity of the permafrost table in this study (data not shown). The biogenic carbon decomposition should be particularly considered when this permafrost unit degraded rapidly. Our data showed a high abundance of type II MOP in the active layer, and the type I Gammaproteobacterial methanotrophs at the permafrost table. Among the type II MOP, the major read abundant groups shift from Xanthobacteraceae $(1.58 \%)$ and Bradyrhizobiaceae $(0.98 \%)$ in the upper of active layer, to Sphingobacteriaceae $(0.62 \%)$ in the deep active layer and to Sphingomonadaceae $(0.27 \%)$ at the permafrost table. This pattern differs from those in cold peatlands where the type II methanotrophs and methylotrophs mainly comprise the members from families of Methylocystaceae and Beijerinckiaceae [35]. On the other hand, most members of type I jump to a peak value of $12.36 \%$ at the permafrost table. Most members belong to the families of Pseudomonadaceae (9.53\%) and Moraxellaceae (2.84\%).

Obviously, the type II members generally decrease with depth, whereas the type I MOP is higher than type II only at the permafrost table $(12.43 \%$ and $1.12 \%$, respectively). The two types of MOP differ in carbon assimilation pathway. Due to this reason, the dynamic of the two MOP groups in the degrading permafrost environments could determine the major players and related major metabolic patterns in different sites and soil layers. Recent thawing of Alaskan permafrost soil was shown to have caused a rapid and dynamic shift of microbial communities involved in cycling of carbon and nitrogen. The type II methanotrophs significantly increased in abundance after thaw, methane previously accumulated in permafrost is released during the thawing process and subsequently consumed by methanotrophic bacteria [52]. The climate warming has accelerated the permafrost degradation and drying of permafrost wetlands in Northeast

\section{References}

1. Jin H, Yu Q, Lü L, Guo D, He R, et al. (2007) Degradation of permafrost in the Xing'anling Mountains, Northeastern China. Permafrost Periglac Process 18: $245-258$.

2. Jin H, Hao J, Chang X, Zhang J, Yu Q et al. (2010) Zonation and assessment of frozen ground conditions for engineering geology along the China-Russia crude oil pipeline route from Mo'he to Daqing, Northeastern China. Cold Reg Sci Technol 64: 213-225.

3. Det Norske Veritas (DNV) (2003) Russia pipeline oil spill study. ESMAP Technical Paper, vol. 034-03. Joint UNDP/World Bank Energy Sector Management Assistance Programme, Norway, 137 p.

4. Snape I, Riddle MJ, Filler DM, Williams PJ (2003) Contaminants in freezing ground and associated ecosystems: key issues at the beginning of the new millennium. Polar Record 39: 291-300.

5. Spormann AM, Widdel F (2000) Metabolism of alkylbenzenes, alkanes, and other hydrocarbons in anaerobic bacteria. Biodegradation 11: 85-105.
China [1]. While thawing, the trapped Gammaproteobacterial MOP is likely to be activated, and trapped organic matter may become more accessible to microbial degradation and subsequently enhance greenhouse gas emissions.

\section{Conclusion}

The 454 pyrosequencing reveals an immense bacterial community in the permafrost soils along the CRCOP in the northern part of Northeast China. These members could be assigned into 39 different phyla, and 223 families or 386 genera. Among them, only five phyla comprise average OTUs abundance more than 5\% across all samples, but they altogether account for $85.08 \%$ of total reads. Proteobacteria (mostly Betaproteobacteria and Alphaproteobacteria) is predominant, making up $41.65 \%$ (6018 OTUs) of the total OTUs identified, followed by Acidobacteria (8.56\%, 1237 OTUs; esp. Acidobacteriaceae), Chloroflexi (8.27\%, 1195 OTUs; esp. Anaerolineaceae), Actinobacteria (7.86\%, 1136 OTUs; esp. Intrasporangiaceae), Bacteroidetes (7.35\%, 1063 OTUs; esp. Sphingobacteria and Flavobacteria). The potential biodegraders are anticipated to be found within the genera of Rhodococcus, Pseudomonas and Sphingomonas which are less than $0.01 \%$ in the indigenous bacterial communities. Moreover, the low abundant groups $(<0.1 \%)$ could account for $69 \%$ and $82 \%$ of total numbers of families and genus observed in this research. Besides, the communities in the upper active layer are relatively more similar to each other than those in the lower active layer and in the permafrost table. The former one seems to be more correlated with the soil TOC, TN, TP, and soil water contents. This study is somewhat weakened in the strength of its conclusions because the lack of biological replicates. However, the results from this study still profile the background bacterial communities among which potential hydrocarbon degraders could be further discovered.

\section{Acknowledgments}

The authors acknowledge Huifang An from Shanghai Major Bio-pharm Biotechnology Co. Ltd. for her technical support in raw data processing. Special thanks are given to Prof. Chien-Lu Ping from University of Alaska Fairbanks (UAF) for improving the language. Great thanks are due to Hanbo Chen, a major developer of $R$ package of VennDiagram for his generous help to complete venn diagram. Finally, the authors wish to express thanks to the insightful comments and suggestions of the anonymous reviewers.

\section{Author Contributions}

Conceived and designed the experiments: SY. Performed the experiments: SY. Analyzed the data: XW SY. Wrote the paper: SY. Rewrote some sections of the manuscript during the revision: HJ QW.

6. Mohn W, Radziminski C, Fortin MC, Reimer K (2001) On site bioremediation of hydrocarbon-contaminated Arctic tundra soils in inoculated biopiles. Appl Microbiol Biotechnol 57: 242-247.

7. Margesin R, Schinner F (2001) Biodegradation and bioremediation of hydrocarbons in extreme environments. Appl Microbiol Biotechnol 56: 650663.

8. Chakraborty R, Coates J (2004) Anaerobic degradation of monoaromatic hydrocarbons. Appl Microbiol Biotechnol 64: 437-446.

9. Atlas RM (1981) Microbial degradation of petroleum hydrocarbons: an environmental perspective. Microbiol Rev 45: 180.

10. Aislabie J, Foght J (2008) Hydrocarbon-degrading bacteria in contaminated cold soils. In: Filler DM, Snape I, Barnes DL, editors. Bioremediation of Petroleum Hydrocarbons in Cold Regions. New York: Cambridge University Press. pp. 69 83 
11. Li G, Huang W, Lerner D, Zhang X (2000) Enrichment of degrading microbes and bioremediation of petrochemical contaminants in polluted soil. Water Research 34: 3845-3853.

12. He Z, Mei B, Wang W, ShengJ, Zhu S, et al. (2003) A pilot test using microbial paraffin-removal technology in Liaohe oilfield. Petroleum Sci Tech 21: 201-210.

13. Wang J, Xu H, Guo S (2007) Isolation and characteristics of a microbial consortium for effectively degrading phenanthrene. Petroleum Science 4: 68-75.

14. Cheng L, Qiu TL, Li X, Wang WD, Deng Y, et al. (2008) Isolation and characterization of Methanoculleus receptaculi sp. nov. from Shengli oil field, China. FEMS Microbiol Lett 285: 65-71.

15. Ye S, Huang L, Li YO, Ding M, Hu Y, et al. (2006) Investigation on bioremediation of oil-polluted wetland at Liaodong Bay in northeast China. Appl Microbiol Biotechnol 71: 543-548.

16. Li H, Yang SZ, Mu BZ, Rong ZF, Zhang J (2006) Molecular analysis of the bacterial community in a continental high-temperature and water-flooded petroleum reservoir. FEMS Microbiol Lett 257: 92-98.

17. Wang J, Ma T, Zhao L, Lv J, Li G, et al. (2008) PCR-DGGE method for analyzing the bacterial community in a high temperature petroleum reservoir. World J Microbiol Biotech 24: 1981-1987.

18. Liu Y, Chen Y, Jin P, Wang X (2009) Bacterial communities in a crude oil gathering and transferring system (China). Anaerobe 15: 214-218.

19. Zhang F, She YH, Ma SS, Hu JM, Banat IM, et al. (2010) Response of microbial community structure to microbial plugging in a mesothermic petroleum reservoir in China. Appl Microbiol Biotechnol 88: 1413-1422.

20. Yu S, Li S, Tang Y, Wu X (2011) Succession of bacterial community along with the removal of heavy crude oil pollutants by multiple biostimulation treatments in the Yellow River Delta, China. J Environ Sci 23: 1533-1543.

21. Zheng C, He J, Wang Y, Wang M, Huang Z (2011) Hydrocarbon degradation and bioemulsifier production by thermophilic Geobacillus pallidus strains. Bioresource Technol 102: 9155-9161.

22. Li P, Sun T, Stagnitti F, Zhang C, Zhang H, et al. (2002) Field-scale bioremediation of soil contaminated with crude oil. Environ Eng Sci 19: 277289.

23. Huang LC YS, Zhang Y, Li YO, Wang XR, Ding D (2007) Microbial remediation and optimization of oil polluted wetlands at Dalian Bay in China. Biomed Environ Sci 20: 414-419.

24. Yang SZ, Jin HJ, Yu SP, Chen YC, Hao JQ et al. (2010) Environmental hazards and contingency plans along the proposed China-Russia Oil Pipeline route, Northeastern China. Cold Reg Sci Technol 64: 271-278.

25. Steven B, Leveille R, Pollard WH, Whyte LG (2006) Microbial ecology and biodiversity in permafrost. Extremophiles 10: 259-267.

26. Janssen PH (2006) Identifying the dominant soil bacterial taxa in libraries of $16 \mathrm{~S}$ rRNA and 16S rRNA genes. Appl Environ Microbiol 72: 1719-1728.

27. Vishnivetskaya TA, Petrova MA, Urbance J, Ponder M, Moyer CL, et al. (2006) Bacterial community in ancient Siberian permafrost as characterized by culture and culture-independent methods. Astrobiology 6: 400-414.

28. Gilichinsky D, Vishnivetskaya T, Petrova M, Spirina E, Mamykin V, et al. (2008) Bacteria in permafrost. In: Margesin R, Schinner F, Marx J C, Gerday C. editors. Psychrophiles: from biodiversity to biotechnology. Berlin: Springer. pp. 83-102.

29. Margesin R, Miteva V (2011) Diversity and ecology of psychrophilic microorganisms. Res Microbiol 162: 346-361.

30. Rothberg JM, Leamon JH (2008) The development and impact of 454 sequencing. Nat Biotechnol 26: 1117-1124.

31. Schloss PD, Westcott SL, Ryabin T, Hall JR, Hartmann M, et al. (2009) Introducing mothur: open-source, platform-independent, community-supported software for describing and comparing microbial communities. Appl Environ Microbiol 75: 7537-7541.
32. Kolde R (2012) pheatmap: Pretty Heatmaps. $R$ package version 061.

33. Chen HB (2012) VennDiagram: Generate high-resolution Venn and Euler plots. $R$ package version 113

34. Oksanen J, Blanchet FG, Kindt R, Legendre P, Minchin PR, et al. (2012) vegan: Community Ecology Package $R$ package version 20-3.

35. Dedysh SN (2011) Cultivating uncultured bacteria from northern wetlands: knowledge gained and remaining gaps. Front Microbiol 2: 184.

36. Wilhelm RC, Niederberger TD, Greer C, Whyte LG (2011) Microbial diversity of active layer and permafrost in an acidic wetland from the Canadian High Arctic. Can J Microbiol 57: 303-315.

37. Sogin ML, Morrison HG, Huber JA, Welch DM, Huse SM, et al. (2006) Microbial diversity in the deep sea and the underexplored "rare biosphere". P Natl Acad Sci USA 103: 12115-12120.

38. Hansel CM, Fendorf S, Jardine PM, Francis CA (2008) Changes in bacterial and archaeal community structure and functional diversity along a geochemically variable soil profile. Appl Environ Microbiol 74: 1620-1633.

39. Liebner S, Harder J, Wagner D (2008) Bacterial diversity and community structure in polygonal tundra soils from Samoylov Island, Lena Delta, Siberia. Int Microbiol 11: 195-202.

40. Wallenstein MD, McMahon S, Schimel J (2007) Bacterial and fungal community structure in Arctic tundra tussock and shrub soils. FEMS Microbiol Ecol 59: 428-435.

41. Aeckersberg F, Rainey FA, Widdel F (1998) Growth, natural relationships, cellular fatty acids and metabolic adaptation of sulfate-reducing bacteria that utilize long-chain alkanes under anoxic conditions. Arch Microbiol 170: 361369.

42. Williams PA, Kay CM (2008) The catabolism of aromatic compounds by Acinetobacter. In: Gerischer U, editor. Acinetobacter Molecular Microbiology. Norfolk: Casiter Academic Press. pp. 99-118.

43. Xun L, Topp E, Orser CS (1992) Diverse substrate range of a Flavobacterium pentachlorophenol hydroxylase and reaction stoichiometries. J Bacteriol 174: 2898-2902.

44. Lo K, Zhu C, Cheuk W (1998) Biodegradation of pentachlorophenol by Flavobacterium species in batch and immobilized continuous reactors. Environ Technol 19: 91-96.

45. Tyagi M, Da Fonseca MM, De Carvalho CG (2011) Bioaugmentation and biostimulation strategies to improve the effectiveness of bioremediation processes. Biodegradation 22: 231-241.

46. Saul DJ, Aislabie JM, Brown CE, Harris L, Foght JM (2005) Hydrocarbon contamination changes the bacterial diversity of soil from around Scott Base, Antarctica. FEMS Microbiol Ecol 53: 141-155.

47. Ai M, Ai M, Li H, Shi R, Han S, et al. (2010) Bacterial community structure in production water from oil reservoirs in Daqing Oilfield. Chinese J Appl Ecol 21:1014-1020 (in Chinese).

48. Gerdes B, Brinkmeyer R, Dieckmann G, Helmke E (2005) Influence of crude oil on changes of bacterial communities in Arctic sea-ice. FEMS Microbiol Ecol 53: 129-139.

49. Aislabie J, Fraser R, Duncan S, Farrell RL (2001) Effects of oil spills on microbial heterotrophs in Antarctic soils. Polar Biol 24: 308-313.

50. Rike A, Børresen M, Instanes A (2001) Response of cold-adapted microbial populations in a permafrost profile to hydrocarbon contaminants. Polar Record 37: 239-248.

51. Niu HG, Ma, X H (1995) Swamps in China. Beijing: The Commercial Press. $143 \mathrm{p}$.

52. Mackelprang R, Waldrop MP, DeAngelis KM, David MM, Chavarria KL, et al. (2011) Metagenomic analysis of a permafrost microbial community reveals a rapid response to thaw. Nature 480: 368-371. 